\title{
Prevalence and Pattern of Depression Among Women of Polygamous and Monogamous Family Settings in Rural Areas of Sokoto State, Nigeria
}

\author{
Habibullah Adamu*, Oche Mansur Oche, Iye Ruth Abel, Kasimu Alhaji Garba, \\ Bashar Bala Zubairu
}

Department of Community Health, Usmanu Danfodiyo University, Sokoto, Nigeria

\section{Email address:}

habibullah.adamu@udusok.edu.ng (H. Adamu), ochedr@hotmail.com (O. M. Oche), iyeabel@gmail.com (I. R. Abel), kasimgarba@gmail.com (K. A. Garba)

${ }^{*}$ Corresponding author

\section{To cite this article:}

Habibullah Adamu, Oche Mansur Oche, Iye Ruth Abel, Kasimu Alhaji Garba, Bashar Bala Zubairu. Prevalence and Pattern of Depression Among Women of Polygamous and Monogamous Family Settings in Rural Areas of Sokoto State, Nigeria. Central African Journal of Public Health. Vol. 7, No. 1, 2021, pp. 11-22. doi: 10.11648/j.cajph.20210701.12

Received: December 6, 2020; Accepted: December 14, 2020; Published: January 15, 2021

\begin{abstract}
World Health Organization recognizes depression as one of the leading mental illnesses of public health concern, with estimated 300 million people affected. This study assessed the prevalence, pattern and risk factors associated with depression among women in rural areas of Sokoto state. A comparative cross-sectional study was carried out and using a multistage sampling technique, 383 respondents (monogamous 193, polygamous 185) were recruited into the study. A set of structured questionnaire was used to collect data which was analyzed using the Statistical Package for Social sciences (SPSS) version 23. The mean age of the respondents was $33.91 \pm 6.8$ years in monogamous setting and $35.27 \pm 8.3$ years in polygamous setting $(\mathrm{t}=1.189, \mathrm{p}=0.235)$. In both settings, majority of the women were married (monogamous $92.2 \%$, polygamous $89.2 \%$, $\mathrm{P}>0.05)$ and all were Muslims. The overall prevalence of depression in both groups was $51.2 \%(\mathrm{n}=197)$. With respect to family type, prevalence was higher among women in monogamous setting $(54.2 \%)$ as compared to those from polygamous setting (49.7\%) ( $\mathrm{P}=0.578)$. In both groups, mild depression was the most common type of depression ( $43.1 \%$ among monogamous vs $54.3 \%$ among polygamous). Depressive symptoms experienced nearly everyday by respondents in both groups include feeling hopeless and down, having difficulty falling asleep and feeling tired. Significant predictors of depression were marital status and feeling sad for a long time. In conclusion, the overall prevalence of depression was high in this study and the prevalence was slightly higher in monogamous group than polygamous; most respondents in both groups had mild depression. Significant predictors of depression include marital status and being sad for long time. There is need for government health agencies and other partners to strengthen mental health services at community levels to enable early detection and proper management of depression among couples.
\end{abstract}

Keywords: Depression, Prevalence, Pattern, Polygamy, Monogamy, Rural Areas

\section{Introduction}

Depression is a mental illness of global concern, though sometimes vague in presentation, its effects are ghastly [1]. It is a broad and heterogeneous disorder characterized by persistently low mood and/or loss of interest in most activities [1]. According to American Psychiatry Association, symptoms of depression can vary from mild to severe and can also include changes in appetite (weight loss or gain unrelated to dieting), trouble in falling asleep or sleeping too much, increased fatigue, suicidal thoughts, increase in purposeless physical activities, slowed movement and speech, feeling worthless or guilty, difficulty in thinking and concentrating or making decisions [2]. Symptoms must last at least two weeks for a diagnosis of depression to be made. Depression can happen at any time but on the average, it first appears during the late teenage to mid-twenties [2]. when long-lasting and with moderate or severe intensity, depression may become a serious health condition [3]. The burden of depression and 
other mental health conditions is on the rise globally. The World Health Organization has ranked depression as the fourth leading cause of disability worldwide [3]. Globally, more than 300 million people of all ages suffer depression. Depression affects an estimated 1 in 15 adults (6.7\%) in any given year and 1 in 6 people will experience depression at some point in their lifetime [3].

Women are more likely to experience depression than men and it has been shown that one third of women will experience a major depressive episode in their lifetime [2, 4, 5]. Factors that play a role in the etiology and clinical manifestation of depression include biochemistry, genetics, personality, environmental factors amongst others. While changes in the level of neurotransmitters in the brain (like acetylcholine, serotonin, dopamine and others) may contribute to symptoms of depression, which have been found to run in some families [2]. Also, people with low self-esteem who are easily overwhelmed by stress, or who are generally pessimistic appear to be more likely to experience depression [2]. Another factor influencing the etiology of depression is the social environment of an individual as continuous exposure to neglect, abuse, violence or poverty might make some people more vulnerable to depression. Worthy of note is the type of family setting the individual belongs (i.e. monogamous and polygamous settings) [6].

Population studies have consistently shown that major depression is about twice as common in women as in men although it is still unclear why this is so [3, 7]. According to a 2015 global health estimate, 86 million (27\%) people suffer depression in Western Pacific Region. In the Americas, a whopping 48.16 million (15\%) people suffer from depression while in the Eastern Mediterranean Region, 26.19 million people $(9 \%)$ suffer depression. An estimated 40.20 million people $(12 \%)$ and 52.98 million people $(16 \%)$ are affected in Europe and Africa respectively [8]. In Turkey, a study on the mental health aspects of Turkish women from polygamous versus monogamous families reported that there was a higher prevalence of depression among married women of polygamous setting than those of monogamous setting [6].

The type of family setting the individual belongs (i.e. monogamous and polygamous settings) has also been linked with depression [3]. Studies conducted in different countries have shown that polygamy can lead to co-wife jealousy, competition and unequal distribution of household and emotional resources, and generate acrimony between co-wives and between the children of different wives. They have also shown that polygamy is associated with mental illnesses (in particular, depression and anxiety) [9, 10]. In women, depression affects career, relationship, social life and sense of self-worth. This has a ripple effect in the family and society at large as mentally disabled women would concomitantly raise unstable children who would be a threat to the society [11].

In Turkey and some countries in the Middle East where monogamous and polygamous marriages are commonly practiced, studies have observed a link between the type of marriage settings women belong to and prevalence and pattern of depression [6]. In Sokoto and indeed Nigeria at large, studies on depression largely focused on its prevalence and pattern among general population; there is no record of any study within the study area that looked at depression among women in relation to the type of marriage setting they are living in (whether monogamous or polygamous), even though both types of marriages are common in the area. This study therefore, comparatively assessed the prevalence and pattern of depression among married women in monogamous and polygamous settings in a rural community of Sokoto state, north-west Nigeria.

\section{Materials and Method}

\subsection{Study Area}

The study was conducted in Bodinga Local Government Area (LGA), one of the 23 LGAs in Sokoto state. It is located about $15 \mathrm{~km}$ away from Sokoto town, the capital of Sokoto State. Bodinga LGA has 11 political wards, including Bodinga/Tauma, Badau/Darhela, Bagarawa, Bangi/Dabaga among others. At growth rate of $3.01 \%$, the population of Bodinga LGA is projected to be 264,832 in 2020 [12]. The major tribes are Hausa, Fulani, other tribes are Yoruba, Igbos etc and there are both Muslims and Christians living there, however, the majority are Muslims. Bodinga LGA has one General hospital, 15 primary schools, 10 secondary schools and one tertiary institution which offers Diploma in Islamic education and Arabic studies [12].

\subsection{Study Design}

It was a comparative cross-sectional study design involving women married in monogamous and polygamous marriage setting.

\subsection{Study Population}

This comprised of previously and currently married women residing in Bodinga Local Government Area of Sokoto state for a period not less than one year prior to the study (Inclusion criteria).

\subsection{Sample Size Determination}

The formula for determining sample size in a comparative study [13] was used to compute the sample size

$$
n=\frac{2\left(Z_{\alpha}+Z_{\beta}\right)^{2} p_{1} q_{1}+p_{2} q_{2}}{\left(p_{1}-p_{2}\right)^{2}}
$$

$\mathrm{Z} \alpha=1.96$

$\mathrm{Z} \beta=0.84$

$\mathrm{P}_{1}=$ prevalence of depression among women in monogamous marriage [14] $=18 \%=0.18$

$\mathrm{P}_{2}=$ prevalence of depression among women in polygamous marriage $[14]=36 \%=0.36$

$\mathrm{q}_{1}=$ complimentary probability for factor under study among women in monogamous marriage

$\left(1-\mathrm{p}_{1}\right)=1-0.18=0.82$ 
$\mathrm{q}_{2}=$ complimentary probability for factor under study among women in polygamous marriage

$$
\begin{gathered}
\left(1-\mathrm{p}_{2}\right)=1-0.36=0.64 \\
\mathrm{n}=\frac{2(1.92+0.84)^{2}(0.18 \times 0.82)+(0.36 \times 0.64)}{(0.18-0.36)^{2}} \\
\mathrm{n}=\frac{2(7.84)(0.1476)+(0.2304)}{(-0.18)^{2}} \\
\mathrm{n}=\frac{15.68 \times 0.378}{0.0324} \\
\mathrm{n}=182.9
\end{gathered}
$$

To adjust for non-response, the minimum sample size " $n$ " was divided by estimated response rate $(95 \%=0.95)$ as follows

$$
\begin{gathered}
\text { Adjusted sample size }=\frac{183}{0.95} \\
=192.63 \\
\approx 193 \text { per group }
\end{gathered}
$$

\subsection{Sampling Technique}

Multistage sampling technique was used to select the study participants as follows:

Stage 1: Out of the three senatorial zones in Sokoto state (Sokoto East, Sokoto Central and Sokoto South), one senatorial zone (Sokoto South) was selected using simple random sampling technique, by balloting procedure.

Stage 2: Out of the seven LGAs in Sokoto south senatorial zone, one LGA (Bodinga LGA) was selected using simple random sampling technique, by balloting procedure.

Stage 3: Bodinga LGA has 11 polical wards and out of these wards, one political ward (Bodinga/Tauma) was selected using simple random sampling technique by balloting procedure.

Stage 4: Five settlements in Bodinga/Tauma ward (namely Bare-Bari, Shiyarsarki, Makera, Shiyarfulani and Yargatari) were selected using simple random sampling technique by balloting procedure.

In each of the selected settlements, house numbering was done with identification of houses that are into monogamous or polygamous marriages, after which proportionate allocation of samples was made for each settlement.

Stage 5: From each of the selected settlement, a central location was identified and with a spin of a bottle, the nearest house was first chosen. Thereafter, consecutive houses were visited to identify eligible households and respondents.

These steps were followed to select respondents in both monogamous and polygamous marriage setting until minimum required sample size in each group was obtained. For respondents married in polygamous setting however, simple random sampling technique by balloting was used to select one woman for the study.

\subsection{Instrument / Method of Data Collection}

Data was collected using a pretested structured questionnaire which was uploaded on open data kit (ODK) version 1.23.2. The questionnaire had three sections with thirty-three stem questions.

Section A: Contained questions on socio-demographic profile of respondents, section B: Contained questions on prevalence and risk factors of depression, section $\mathrm{C}$ : Contained questions on pattern of depression which was adapted from the Patient Health Questionnaire version nine (PHQ-9), a standardized multipurpose instrument for screening, monitoring and measuring the severity of depression [15].

The questionnaire was administered to each eligible respondent at home, after seeking for their consent; the data collection lasted for a period of eight days.

\subsection{Personnel/Training}

Thirteen research assistants comprising of three 600 level medical students ( 2 males and 1 female student) and ten 300 level medical students (all females) were trained by the researcher. The training was for a period of two days and it covered general overview of the family social group, depression, questionnaire/survey instruments, sampling techniques, field activities, ethics of fieldwork, general principles of research, interpersonal communication skills and the use of ODK for data collection.

\subsection{Pretest}

The questionnaire (uploaded on ODK) was pretested on 40 married women (20 in monogamous marriage and 20 in polygamous) selected from Kalambaina town in Wamakko LGA of Sokoto state. This allowed for further assessment and modification of the study instruments and the conduct of the study.

\subsection{Data Analysis}

Data was exported from the ODK server to Microsoft excel 2016, and transferred to IBM SPSS version 20 for analysis. Continuous variables were summarized as mean and standard deviation (SD) while categorical variables were presented as frequencies and percentages. Chi-square test was used to test the significance of association between categorical variables while logistic regression was used to determine the predictors of depression. Results were presented in tables and figures; level of significance was set at $5 \%(\mathrm{p}<0.05)$.

\subsection{Ethical Consideration}

Ethical approval was obtained from Sokoto State Ministry of Health Research Ethics Committee (HREC). Individual informed verbal consent was also obtained from the respondents before the questionnaire was administered, all data obtained were handled with utmost confidentiality. 


\section{Results}

Out of 386 questionnaires that were administered, 6 questionnaires were removed because the interviews were not completed, thus, 378 forms were analyzed giving 97.9\% response rate.

The ages of respondents in both the monogamous and polygamous groups ranged from 17 to 76 years. The mean age of the respondents in the monogamous group was $33.91 \pm 6.8$, while that of respondents in the polygamous group was $35.27 \pm 8.3$, there was no significant difference in the mean age of the respondents in both groups $(\mathrm{t}=1.189, \mathrm{p}=0.235)$. Those within 20-29 year age group constituted the highest proportion of respondents in both groups (monogamous 38.3\%, polygamous $32.4 \%$ ). There was no statistically significant difference in the age distribution of respondents in the two groups $\left(\mathrm{X}^{2}=2.278, \mathrm{P}=0.890\right)$. Majority of the respondents in both groups were married (monogamous $92.2 \%$, polygamous $89.2 \%)$. All the respondents in both groups (100\%) were Muslims and in terms of their distribution by tribe, there were more respondents from the Hausa tribe in the polygamous group $(90.3 \%)$ than in the monogamous group $(81.9 \%)$; the difference in their distribution was statistically significant $\left(\mathrm{X}^{2}=6.08, \mathrm{P}=0.033\right)$. More than half of the respondents in both monogamous and polygamous groups had no formal education $[109 \quad(56.5 \%)$ and $107 \quad(57.8 \%)$ respectively, $\mathrm{p}=0.227$ ] and in terms of their occupation, petty trading constituted the highest proportion in both groups [monogamous $114(59.1 \%)$, polygamous $97(52.4 \%)$ ]. (Table 1).

Table 1. Socio-demographic characteristics of respondents in rural areas of Sokoto State.

\begin{tabular}{|c|c|c|c|}
\hline \multirow{2}{*}{ Variable } & \multicolumn{2}{|l|}{ Type of family setting } & \multirow{2}{*}{ Test statistic } \\
\hline & Monogamous N=193 (\%) & Polygamous $\mathrm{N}=185(\%)$ & \\
\hline \multicolumn{4}{|l|}{ Age (in years) } \\
\hline$<20$ & $6(3.1)$ & $9(4.9)$ & \multirow{7}{*}{$\begin{array}{l}X^{2}=2.278 \\
P=0.890\end{array}$} \\
\hline $20-29$ & $74(38.3)$ & $60(32.4)$ & \\
\hline $30-39$ & $46(23.8)$ & $49(26.5)$ & \\
\hline $40-49$ & $28(14.5)$ & $31(16.8)$ & \\
\hline $50-59$ & $25(13.0)$ & $23(12.4)$ & \\
\hline $60-69$ & $10(5.2)$ & $9(4.9)$ & \\
\hline $70-79$ & $4(2.1)$ & $4(2.2)$ & \\
\hline \multicolumn{3}{|l|}{ Marital status } & \multirow{4}{*}{$\begin{array}{l}X^{2}=1.081 \\
P=0.582\end{array}$} \\
\hline Married & $178(92.2)$ & $165(89.2)$ & \\
\hline Widowed & $10(5.2)$ & $14(7.6)$ & \\
\hline Separated & $5(2.6)$ & $6(3.2)$ & \\
\hline \multicolumn{3}{|l|}{ Tribe } & \multirow{4}{*}{$\begin{array}{l}X^{2}=6.08 \\
P=0.033\end{array}$} \\
\hline Hausa & $158(81.9)$ & $167(90.3)$ & \\
\hline Fulani & $30(15.5)$ & $13(7.0)$ & \\
\hline Others & $5(2.6)$ & $5(2.7)$ & \\
\hline \multicolumn{3}{|l|}{ Religion } & \multirow{4}{*}{ N/A } \\
\hline Islam & $193(100)$ & $185(100)$ & \\
\hline Christianity & 0 & 0 & \\
\hline Others & 0 & 0 & \\
\hline \multicolumn{3}{|l|}{ Educational status of respondents } & \multirow{5}{*}{$\begin{array}{l}X^{2}=4.340 \\
P=0.227\end{array}$} \\
\hline No formal education & $109(56.5)$ & $107(57.8)$ & \\
\hline Primary school certificate & $32(16.6)$ & $40(21.6)$ & \\
\hline Secondary school certificate & $48(24.9)$ & $32(17.3)$ & \\
\hline Tertiary certificate & $4(2.1)$ & $6(3.2)$ & \\
\hline \multicolumn{3}{|c|}{ Educational status of respondent's husband } & \multirow{5}{*}{$\begin{array}{l}X^{2}=3.731 \\
P=0.292\end{array}$} \\
\hline No formal education & $42(21.8)$ & $55(29.7)$ & \\
\hline Primary school certificate & $29(15.0)$ & $24(13.0)$ & \\
\hline Secondary school certificate & $38(19.7)$ & $38(20.5)$ & \\
\hline Tertiary certificate & $84(43.5)$ & $68(36.8)$ & \\
\hline \multicolumn{3}{|l|}{ Occupation of respondents } & \multirow{6}{*}{$\begin{array}{l}X^{2}=5.767 \\
P=0.217\end{array}$} \\
\hline Housewife & $45(23.3)$ & $63(34.1)$ & \\
\hline Petty trader & $114(59.1)$ & $97(52.4)$ & \\
\hline Tailor & $13(6.7)$ & $12(6.5)$ & \\
\hline Civil servant & $3(1.6)$ & $2(1.1)$ & \\
\hline Others & $17(8.8)$ & $11(5.9)$ & \\
\hline \multicolumn{3}{|c|}{ Occupation of respondents' husband } & \multirow{8}{*}{$\begin{array}{l}X^{2}=14.768 \\
P=0.022\end{array}$} \\
\hline Farmer & $14(7.3)$ & $25(13.5)$ & \\
\hline Trader & $58(30.1)$ & $48(26.0)$ & \\
\hline Artisan/Vocational worker & $17(8.8)$ & $27(14.6)$ & \\
\hline Civil Servant & $60(31.1)$ & $59(31.9)$ & \\
\hline Law enforcement agent & $1(0.5)$ & $4(2.2)$ & \\
\hline Unemployed & $11(5.7)$ & $6(3.2)$ & \\
\hline Others & $32(16.6)$ & $16(8.6)$ & \\
\hline Duration of marriage (in years) & & & $X^{2}=2.153$ \\
\hline $0-10$ & $74(38.3)$ & $67(36.2)$ & $\mathrm{P}=0.905$ \\
\hline
\end{tabular}




\begin{tabular}{|c|c|c|c|}
\hline \multirow{2}{*}{ Variable } & \multicolumn{2}{|l|}{ Type of family setting } & \multirow{8}{*}{ Test statistic } \\
\hline & Monogamous N=193 (\%) & Polygamous N=185 (\%) & \\
\hline $11-20$ & $54(30.0)$ & $50(27.0)$ & \\
\hline $21-30$ & $31(16.1)$ & $31(16.8)$ & \\
\hline $31-40$ & $27(14.0)$ & $25(13.5)$ & \\
\hline $41-50$ & $3(1.6)$ & $7(3.8)$ & \\
\hline $51-60$ & $3(1.6)$ & $4(2.2)$ & \\
\hline $61-70$ & $1(0.5)$ & $1(0.5)$ & \\
\hline
\end{tabular}

Overall prevalence of depression in both groups was $52.1 \%(\mathrm{~N}=197)$, of which $28.0 \%(\mathrm{~N}=106)$ was among monogamous and $24.1 \%(\mathrm{~N}=91)$ among polygamous group. There was no statistically significant difference between the two groups $\left(\mathrm{X}^{2}=0.788\right.$, $\mathrm{p}=0.375)$ [Figure 1].

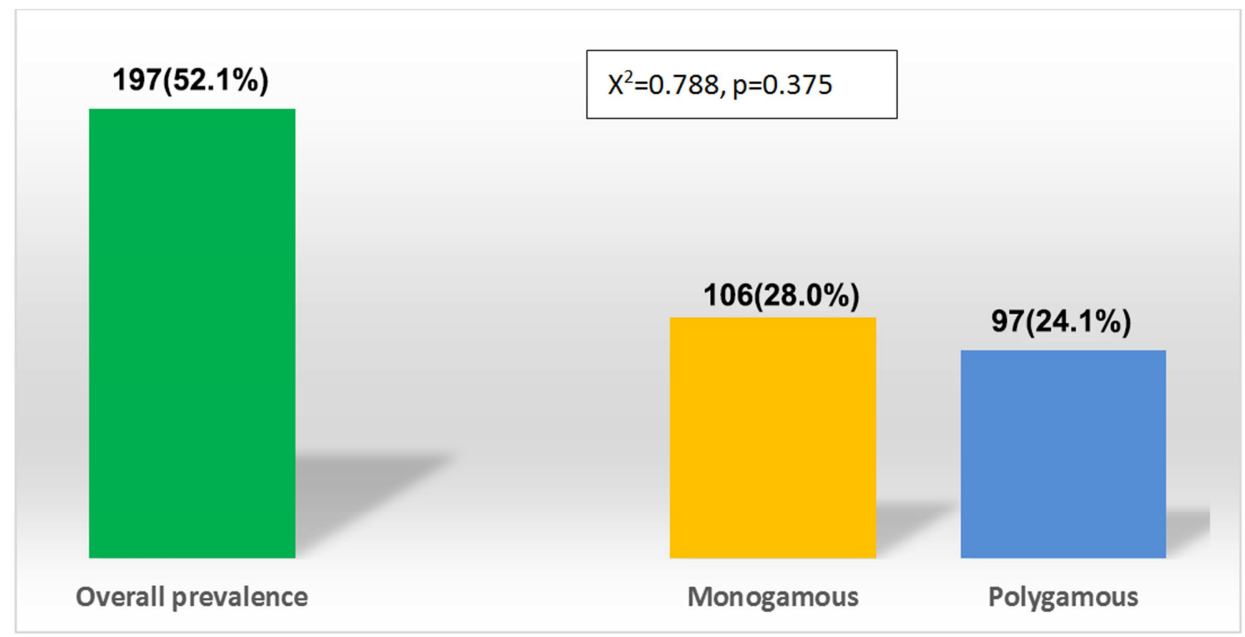

Figure 1. Overall prevalence of depression, prevalence of depression in monogamous and polygamous marriages among the respondents in rural areas of Sokoto State.

Of the 52.1\% respondents with depression in both groups, 95 (25.2\%) had mild depression, 56 (14.8\%) had moderate depression while $14(3.6 \%)$ had severe depression (Figure 2).

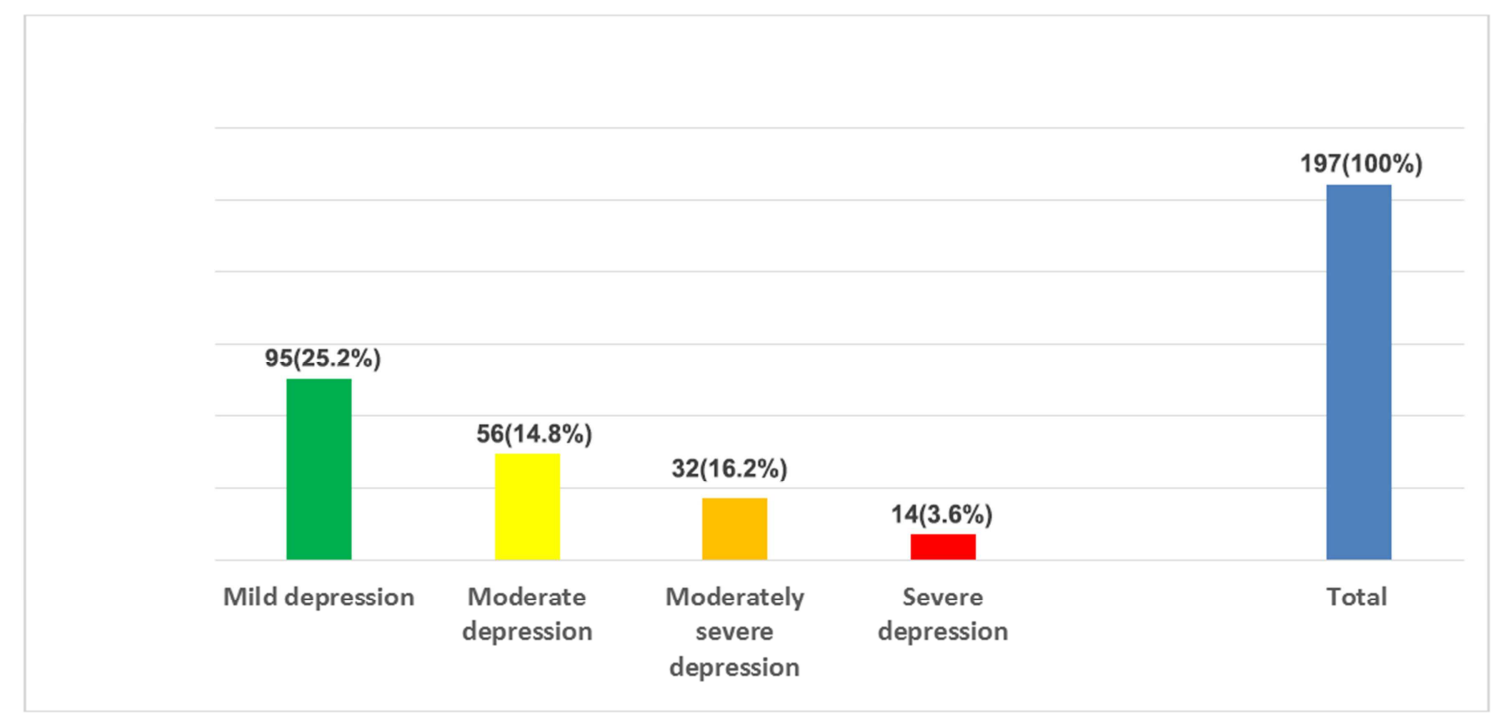

Figure 2. Prevalence of the different types of depression among respondents in rural areas of Sokoto State.

Regarding types of depression according to marriage setting, $46(43.1 \%)$ and $49(54.3 \%)$ of respondents in monogamous and polygamous groups respectively had mild depression, $37(34.9 \%)$ in monogamous and $20(21.3 \%)$ in polygamous group had moderate depression whereas $5(4.6 \%)$ in monogamous and $9(9.6 \%)$ in polygamous group had severe depression. There was no statistically significant difference in the distribution depression by type according to marriage setting $\left(\mathrm{X}^{2}=7.336, \mathrm{p}=0.119\right)$ [Figure 3]. 


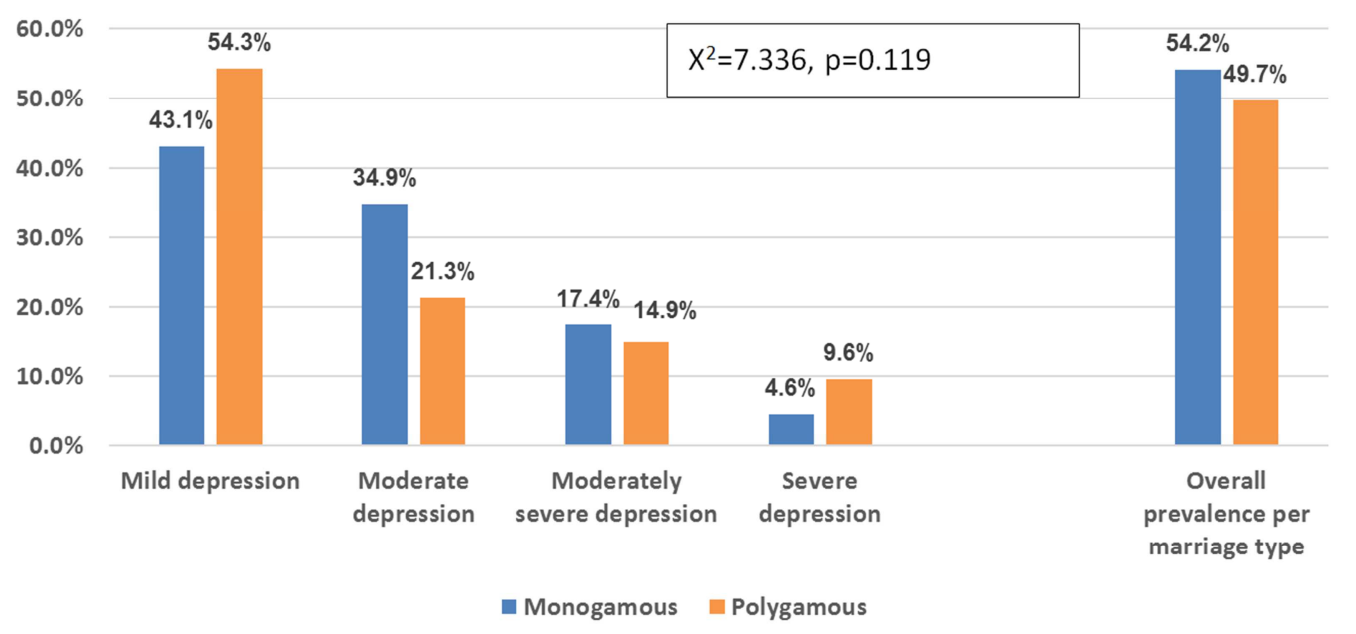

Figure 3. Prevalence and distribution of types of depression according to marriage setting among respondents in rural areas of Sokoto State.

Depressive symptoms experienced nearly everyday by respondents included feeling hopeless and down [monogamous $33(16.6 \%)$ vs. polygamous $18(9.6 \%)$ ]; having difficulty falling asleep [monogamous $32 \quad(15.9 \%)$ vs polygamous $26(13.8 \%)$ ]; feeling tired [monogamous 56 $(28.0 \%)$ vs polygamous $42(22.2 \%)$ ] and having trouble concentrating on things like watching TV [monogamous 27 $(13.5 \%)$ vs polygamous $24(12.8 \%)$ ]; however, there was no statistically significant difference in their distribution between the two groups ( $>0.05$ ). The only depressive symptom that was significantly associated with type of marriage setting was loss of appetite; up to $21(10.4 \%)$ of respondents in monogamous marriage setting said they had loss of appetite for at least seven days within the past two weeks whereas in the polygamous group only $5(2.6 \%)$ loss of appetite $\left(\mathrm{X}^{2}=12.419, \mathrm{P}=0.006\right)$. [Table 2]

Table 2. Pattern of depression among respondents in rural areas of Sokoto State.

\begin{tabular}{|c|c|c|c|}
\hline \multirow{2}{*}{ Variable } & \multicolumn{2}{|l|}{ Type of family setting } & \multirow{2}{*}{ Test statistic $\left(\mathrm{X}^{2}\right)$} \\
\hline & Monogamous setting (\%) & Polygamous setting (\%) & \\
\hline \multicolumn{4}{|l|}{ Having little interest in the things you used to do before } \\
\hline Not at all & $110(55.0)$ & $106(56.7)$ & \multirow{4}{*}{$\begin{array}{l}X^{2}=1.142 \\
P=0.765\end{array}$} \\
\hline Rarely & $43(21.5)$ & $45(24.1)$ & \\
\hline About seven days & $13(6.5)$ & $10(5.3)$ & \\
\hline Nearly everyday & $34(17.0)$ & $26(13.9)$ & \\
\hline \multicolumn{4}{|l|}{ Feeling hopeless and down } \\
\hline Not at all & $108(54.3)$ & $110(58.5)$ & \multirow{4}{*}{$\begin{array}{l}X^{2}=6.050 \\
P=0.111\end{array}$} \\
\hline Rarely & $40(20.1)$ & $48(25.5)$ & \\
\hline About seven days & $18(9.0)$ & $12(6.4)$ & \\
\hline Nearly everyday & $33(16.6)$ & $18(9.6)$ & \\
\hline \multicolumn{4}{|l|}{ Having trouble falling asleep or sleep too much } \\
\hline Not at all & $123(61.2)$ & $108(57.4)$ & \multirow{4}{*}{$\begin{array}{l}\mathrm{X}^{2}=1.803 \\
\mathrm{P}=0.619\end{array}$} \\
\hline Rarely & $34(16.9)$ & $40(21.3)$ & \\
\hline About seven days & $12(6.0)$ & $14(7.4)$ & \\
\hline Nearly everyday & $32(15.9)$ & $26(13.8)$ & \\
\hline \multicolumn{4}{|l|}{ Having poor appetite or eat slowly } \\
\hline Not at all & $108(53.7)$ & $126(66.7)$ & \multirow{4}{*}{$\begin{array}{l}X^{2}=12.419 \\
P=0.006\end{array}$} \\
\hline Rarely & $36(17.9)$ & $28(14.8)$ & \\
\hline About seven days & $21(10.4)$ & $5(2.6)$ & \\
\hline Nearly everyday & $36(17.9)$ & $30(15.9)$ & \\
\hline \multicolumn{4}{|l|}{ Feeling tired } \\
\hline Not at all & $91(45.5)$ & $81(42.9)$ & \multirow{4}{*}{$\begin{array}{l}X^{2}=3.789 \\
P=0.287\end{array}$} \\
\hline Rarely & $38(19.0)$ & $49(25.9)$ & \\
\hline About seven days & $15(7.5)$ & $17(53.1)$ & \\
\hline Nearly everyday & $56(28.0)$ & $42(22.2)$ & \\
\hline \multicolumn{4}{|l|}{ Feeling like a failure } \\
\hline Not at all & $145(72.1)$ & $122(64.9)$ & \multirow{4}{*}{$\begin{array}{l}X^{2}=2.429 \\
P=0.496\end{array}$} \\
\hline Rarely & $30(14.9)$ & $35(18.6)$ & \\
\hline About seven days & $6(3.0)$ & $8(4.3)$ & \\
\hline Nearly everyday & $20(10.0)$ & $23(12.2)$ & \\
\hline Having trouble concentrating on things like watching $\mathrm{T}$. $\mathrm{V}$ etc & & & $X^{2}=5.627$ \\
\hline Not at all & $128(64.0)$ & $102(54.3)$ & $\mathrm{P}=0.133$ \\
\hline
\end{tabular}




\begin{tabular}{|c|c|c|c|}
\hline \multirow{2}{*}{ Variable } & \multicolumn{2}{|l|}{ Type of family setting } & \multirow{2}{*}{ Test statistic $\left(\mathrm{X}^{2}\right)$} \\
\hline & Monogamous setting (\%) & Polygamous setting (\%) & \\
\hline Rarely & $34(41.0)$ & $49(26.1)$ & \\
\hline About seven days & $11(17.0)$ & $13(6.9)$ & \\
\hline Nearly everyday & $27(13.5)$ & $24(12.8)$ & \\
\hline \multicolumn{4}{|c|}{ Move or speak slowly } \\
\hline Not at all & $135(67.5)$ & $138(73.0)$ & \multirow{4}{*}{$\begin{array}{l}X^{2}=3.714 \\
0.301\end{array}$} \\
\hline Rarely & $28(14.0)$ & $28(14.8)$ & \\
\hline About seven days & $9(4.5)$ & $8(4.2)$ & \\
\hline Nearly everyday & $28(14.0)$ & $15(7.9)$ & \\
\hline \multicolumn{4}{|c|}{ Thinking about commiting suicide?? } \\
\hline Not at all & $173(86.1)$ & $163(86.2)$ & \multirow{4}{*}{$\begin{array}{l}X^{2}=3.320 \\
P=0.355\end{array}$} \\
\hline Rarely & $11(5.5)$ & $13(6.9)$ & \\
\hline About seven days & $8(4.0)$ & $10(5.3)$ & \\
\hline Nearly everyday & $9(4.5)$ & $3(1.6)$ & \\
\hline
\end{tabular}

Regarding reasons for sadness among respondents, 3 (4.1\%) of those in the monogamous group said the reason for their sadness was because their husbands refused to take care of the kids but in the polygamous group up to $16(21.9 \%)$ said they were sad for the same reason $\left(X^{2}=6.146, p=0.015\right)$. Other reasons for sadness expressed by respondents include husband having affair with another woman [monogamous 1 (1.4\%) vs polygamous $2(2.7 \%) ; p=1.000]$, husband prioritizing co-wife [monogamous $0(0)$ vs polygamous $11(15.1 \%) ; \mathrm{p}=0.005$ ], and husband not satisfying wife sexually [monogamous $2(2.7 \%)$ vs polygamous $2(2.7 \%) ; \mathrm{p}=1.000$ ] (Table 3$)$.

Table 3. Reasons for sadness among respondents in rural areas of Sokoto State.

\begin{tabular}{|c|c|c|c|}
\hline \multirow{2}{*}{ Variable } & \multicolumn{2}{|c|}{ Type of marriage } & \multirow{2}{*}{ Test statistic } \\
\hline & Monogamy & Polygamy & \\
\hline My husband refused to take care of my kids & $3(4.1)$ & $16(21.9)$ & $\mathrm{X}^{2}=6.146, \mathrm{p}=0.015$ \\
\hline My husband was having affair with another woman & $1(1.4)$ & $2(2.7)$ & $\mathrm{p}=1.000$ Fisher's exact \\
\hline My husband prioritized my co-wife & $0(0)$ & $11(15.1)$ & $\mathrm{X}^{2}=8.536, \mathrm{p}=0.005$ \\
\hline My husband does not love me anymore & $1(1.5)$ & $8(11.0)$ & $\mathrm{X}^{2}=3.510, \mathrm{p}=0.078$ \\
\hline My husband beats me & $0(0)$ & $1(1.4)$ & $\mathrm{P}=1.000$ Fisher's exact \\
\hline Husband does not satisfy me sexually & $2(2.7)$ & $2(2.7)$ & $\mathrm{X}^{2}=0.187, \mathrm{P}=1.000$ \\
\hline
\end{tabular}

The highest prevalence of depression among the women in monogamous setting was found within the age group 40-49 years $(60.7 \%)$ while the highest for women in polygamous setting was within the age groups $60-69$ years $(77.8 \%)$; there was however, no statistically significant association between depression and age in both settings $(\mathrm{P}=0.155)$. There was statistically significant relationship between marital status and depression among women married in polygamous setting $\left(\mathrm{X}^{2}=10.837 \mathrm{P}=0.002\right)$. [Table 4]

Table 4. Factors associated with depression in both groups.

\begin{tabular}{|c|c|c|c|c|}
\hline \multirow{3}{*}{ Variable } & \multicolumn{4}{|c|}{ Type of family setting } \\
\hline & \multicolumn{2}{|l|}{ Monogamy } & \multicolumn{2}{|l|}{ Polygamy } \\
\hline & Depression & No depression & Depression & No depression \\
\hline \multicolumn{5}{|l|}{ Age in years } \\
\hline$<40$ & $65(51.6)$ & $61(48.4)$ & $59(50.0)$ & $59(50.0)$ \\
\hline$\geq 40$ & $\begin{array}{l}38(56.7) \\
X^{2}=0.462\end{array}$ & $\begin{array}{l}29(43.3) \\
\mathrm{P}=0.542\end{array}$ & $\begin{array}{l}35(52.2) \\
X^{2}=0.086\end{array}$ & $\begin{array}{l}32(47.8) \\
\mathrm{P}=0.878\end{array}$ \\
\hline \multicolumn{5}{|l|}{ Marital Status } \\
\hline Married & $92(51.7)$ & $86(48.3)$ & $78(46.4)$ & $90(53.6)$ \\
\hline Widowed & $7(70.0)$ & $3(30.0)$ & $12(75.0)$ & $4(25.0)$ \\
\hline Separated & $\begin{array}{l}4(80.0) \\
X^{2}=2.739\end{array}$ & $\begin{array}{l}1(20.0) \\
\mathrm{P}=0.281\end{array}$ & $\begin{array}{l}6(100.0) \\
X^{2}=10.837\end{array}$ & $\begin{array}{l}0(0.0) \\
\mathrm{P}=0.002\end{array}$ \\
\hline \multicolumn{5}{|l|}{ Tribe } \\
\hline Hausa & $83(52.5)$ & $75(47.5)$ & $88(51.8)$ & $82(48.2)$ \\
\hline Fulani & $17(56.7)$ & $13(43.3)$ & $4(26.7)$ & $11(73.3)$ \\
\hline Others & $\begin{array}{l}3(60.0) \\
X^{2}=0.264\end{array}$ & $\begin{array}{l}2(40.0) \\
\mathrm{P}=0.898\end{array}$ & $\begin{array}{l}4(80.0) \\
X^{2}=5.258\end{array}$ & $\begin{array}{l}1(20.0) \\
\mathrm{P}=0.090\end{array}$ \\
\hline \multicolumn{5}{|l|}{ Religion } \\
\hline Islam & $103(53.4)$ & $90(46.6)$ & $96(50.5)$ & $94(49.5)$ \\
\hline Christianity & $0(0)$ & $0(0)$ & $0(0)$ & $0(0)$ \\
\hline Respondent Education & & & & \\
\hline No formal education & $58(53.2)$ & $51(46.8)$ & $56(51.4)$ & $53(48.6)$ \\
\hline Formal education & $45(53.6)$ & $39(46.4)$ & $40(49.4)$ & $41(50.6)$ \\
\hline & $\mathrm{X}^{2}=0.002$ & $\mathrm{P}=1.000$ & $X^{2}=0.074$ & $\mathrm{P}=0.883$ \\
\hline Respondents' husband education & & & & \\
\hline
\end{tabular}




\begin{tabular}{|c|c|c|c|c|}
\hline \multirow{3}{*}{ Variable } & \multicolumn{4}{|c|}{ Type of family setting } \\
\hline & \multicolumn{2}{|c|}{ Monogamy } & \multicolumn{2}{|l|}{ Polygamy } \\
\hline & Depression & No depression & Depression & No depression \\
\hline No formal education & $18(42.9)$ & $24(57.1)$ & $27(48.2)$ & $27(51.8)$ \\
\hline \multirow[t]{2}{*}{ Formal education } & $85(56.3)$ & $66(43.7)$ & $68(51.5)$ & $64(48.5)$ \\
\hline & $X^{2}=2.383$ & $\mathrm{P}=0.162$ & $X^{2}=0.035$ & $\mathrm{P}=0.873$ \\
\hline \multicolumn{5}{|c|}{ Occupation of respondent } \\
\hline Housewife & $24(53.3)$ & $21(46.7)$ & $28(43.8)$ & $36(56.2)$ \\
\hline Petty trader & $64(56.1)$ & $50(43.9)$ & $51(51.5)$ & $48(48.5)$ \\
\hline Tailor & $5(38.5)$ & $8(61.5)$ & $6(46.2)$ & $7(52.8)$ \\
\hline Civil servant & $1(33.3)$ & $2(66.7)$ & $1(50.0)$ & $1(50.0)$ \\
\hline \multirow[t]{2}{*}{ Others } & $8(47.1)$ & $9(52.9)$ & $10(83.3)$ & $2(16.7)$ \\
\hline & $X^{2}=2.263$ & $\mathrm{P}=0.691$ & $\mathrm{X}^{2}=6.481$ & $\mathrm{P}=0.154$ \\
\hline \multicolumn{5}{|c|}{ Occupation of respondent husband } \\
\hline Employed & $98(51.0)$ & $84(49.0)$ & $91(52.6)$ & $82(47.4)$ \\
\hline \multirow[t]{2}{*}{ Unemployed } & $5(45.5)$ & $6(54.5)$ & $4(66.7)$ & $2(33.3)$ \\
\hline & $X^{2}=0.294$ & $\mathrm{P}=0.758$ & $X^{2}=0.461$ & $\mathrm{P}=0.686$ \\
\hline \multicolumn{5}{|c|}{ Duration of marriage in years } \\
\hline$\leq 30$ & $86(54.1)$ & $73(45.9)$ & $73(45.6)$ & $87(54.4)$ \\
\hline \multirow[t]{2}{*}{$>30$} & $17(50.0)$ & $17(50.0)$ & $22(59.5)$ & $15(40.5)$ \\
\hline & $\mathrm{X}^{2}=0.188$ & $\mathrm{P}=0.708$ & $\mathrm{X}^{2}=2.304$ & $\mathrm{P}=0.147$ \\
\hline
\end{tabular}

Being sad for a long period was significantly associated with depression in both groups $(\mathrm{p}<0.001)$ and having family history of depression ( $\mathrm{p}=0.004$ and $\mathrm{p}=0.032$ respectively). Other factors such as sociodemographic factors, smoking cigarette and consumption of alcohol were not found to have any statistically significant association with prevalence of depression among respondents $(\mathrm{p}>0.05)$. [Table 5]

Table 5. Factors associated with depression in both groups.

\begin{tabular}{|c|c|c|c|c|}
\hline \multirow{3}{*}{ Variable } & \multicolumn{4}{|c|}{ Type of family setting } \\
\hline & \multicolumn{2}{|l|}{ Monogamy } & \multicolumn{2}{|l|}{ Polygamy } \\
\hline & Depressed & Not Depressed & Depressed & Not Depressed \\
\hline \multicolumn{5}{|c|}{ Have you been sad for a long period of time that no one could help you? } \\
\hline Yes & $71(68.9)$ & $32(31.1)$ & $56(68.3)$ & $26(31.7)$ \\
\hline \multirow[t]{2}{*}{ No } & $31(35.2)$ & $57(64.8)$ & $40(37.0)$ & $68(63.0)$ \\
\hline & $X^{2}=21.64$ & $\mathrm{P}<0.001$ & $\mathrm{X}^{2}=18.216$ & $\mathrm{P}<0.001$ \\
\hline \multicolumn{5}{|c|}{ Do you smoke cigarettes? } \\
\hline Yes & $3(100.0)$ & $0(0.00)$ & $4(100.0)$ & $0(0.00)$ \\
\hline \multirow[t]{2}{*}{ No } & $99(53.0)$ & $87(46.8)$ & $91(49.2)$ & $94(50.8)$ \\
\hline & $X^{2}=2.600$ & $\mathrm{P}=0.251$ & $X^{2}=4.043$ & $\mathrm{P}=0.121$ \\
\hline \multicolumn{5}{|c|}{ Do you drink alcohol? } \\
\hline Yes & $0(0.00)$ & $0(0.00)$ & $1(100.0)$ & $0(0.00)$ \\
\hline \multirow[t]{2}{*}{ No } & $103(53.9)$ & $88(46.1)$ & $94(50.3)$ & $93(49.7)$ \\
\hline & N/A & & $X^{2}=0.984$ & $\mathrm{P}=1.000$ \\
\hline \multicolumn{5}{|c|}{ Does your husband smoke cigarettes? } \\
\hline Yes & $27(75.0)$ & $9(25.0)$ & $22(66.7)$ & $11(33.3)$ \\
\hline \multirow[t]{2}{*}{ No } & $76(48.4)$ & $81(51.6)$ & $72(46.5)$ & $83(53.5)$ \\
\hline & $X^{2}=8.321$ & $\mathrm{P}=0.005$ & $X^{2}=4.447$ & $\mathrm{P}=0.054$ \\
\hline \multicolumn{5}{|c|}{ Does your husband drink alcohol? } \\
\hline Yes & $0(0.00)$ & $0(0.00)$ & $2(100.0)$ & $0(0.00)$ \\
\hline \multirow[t]{2}{*}{ No } & $103(53.4)$ & $90(46.6)$ & $92(49.5)$ & $94(50.5)$ \\
\hline & N/A & & $\mathrm{X}^{2}=2.022$ & $\mathrm{P}=0.497$ \\
\hline \multicolumn{5}{|c|}{ Any family history of mental illness? } \\
\hline Yes & $32(72.7)$ & $12(27.3)$ & $22(68.8)$ & $10(13.2)$ \\
\hline \multirow[t]{2}{*}{ No } & $70(47.6)$ & $77(52.4)$ & $74(46.8)$ & $84(53.2)$ \\
\hline & $X^{2}=8.579$ & $\mathrm{P}=0.004$ & $X^{2}=5.112$ & $\mathrm{P}=0.032$ \\
\hline
\end{tabular}

Predictors of depression in both groups were marital status [monogamous $(\mathrm{OR}=0.229, \mathrm{p}=0.024,95 \% \mathrm{CI}=0.064-0.823)$ Vs. polygamous $(\mathrm{OR}=0.252, \mathrm{p}=0.009,95 \% \mathrm{CI}=0.089-0.714)]$, being sad for long time [monogamous $(\mathrm{OR}=3.427, \mathrm{p}<0.001$, $95 \% \mathrm{CI}=1.766-6.649)$ Vs. polygamous $(\mathrm{OR}=3.818, \mathrm{p}<0.001$,
95\% $\mathrm{CI}=1.900-7.671)]$ and having loss of interest in doing things as previously done [monogamous ( $\mathrm{OR}=17.462$, $\mathrm{p}<0.001, \quad 95 \% \quad \mathrm{CI}=5.088-59.929) \quad$ Vs. $\quad$ polygamous $(\mathrm{OR}=21.599, \mathrm{p}<0.001,95 \% \mathrm{CI}=4.818-96.820)]$ (Table 6). 
Table 6. Predictors of depression among respondents married in monogamous and polygamous setting.

\begin{tabular}{|c|c|c|c|c|c|c|c|c|}
\hline \multirow{3}{*}{ Predictor } & \multicolumn{4}{|c|}{ Monogamous } & \multicolumn{4}{|c|}{ Polygamous } \\
\hline & \multirow[t]{2}{*}{ aOR } & \multicolumn{2}{|c|}{$95 \%$ CI } & \multirow[t]{2}{*}{ p value } & \multirow[t]{2}{*}{ aOR } & \multicolumn{2}{|c|}{$95 \% \mathrm{CI}$} & \multirow[t]{2}{*}{ p value } \\
\hline & & lower & upper & & & lower & upper & \\
\hline Being very sad for a long time & 3.427 & 1.766 & 6.649 & $<0.001$ & 3.818 & 1.900 & 7.671 & $<0.001$ \\
\hline Family history of mental illness & 1.930 & 0.774 & 4.814 & 0.158 & 1.657 & 0.708 & 3.877 & 0.254 \\
\hline $\begin{array}{l}\text { History of loss of interest in doing things as previously done } \\
\mathrm{aOR}=\text { adjusted Odds Ratio } \mathrm{CI}=\text { Confidence Interval } *=\text { Referen }\end{array}$ & $\begin{array}{l}17.462 \\
\text { e group }\end{array}$ & 5.088 & 59.929 & $<0.001$ & 21.599 & 4.818 & 96.820 & $<0.001$ \\
\hline
\end{tabular}

\section{Discussion}

This study was conducted among women who were ever married either in polygamous and monogamous setting in Bodinga Local Government Area of Sokoto State, to determine the prevalence, pattern, and risk factors associated with depression among women in the study area.

In this study, respondents with the highest proportion were those within 20 to 29 years age group in both monogamous (38.3\%) and polygamous settings (32.4\%); with mean ages of $33.91+/ 6.8$ years for women in monogamous setting and $35.27+/-8.3$ for women in polygamous setting. This finding is similar to the findings of a study by Ozkan et al on mental health aspects of Turkish women married in polygamous versus monogamous settings, which also reported that those aged between 20 to 29 years constituted the highest proportion of their respondents [6]. These findings may be attributed to the fact that most marriages among women occur within this age group. More than half of the respondents in both groups had no formal education [109 (56.5\%) vs 107 (57.8\%)] for both women in polygamous and monogamous settings. This is not surprising because in Sokoto state, up to $88.4 \%$ of women have no education and only $3.2 \%$ have educational attainment beyond secondary school; this is the lowest in the country [16].

Furthermore, husbands who had no formal education in monogamous setting constituted $(21.8 \%)$ as compared to those in polygamous settings $(29.7 \%)$. This is in contrast to the findings of a study by Al-Krenawi et al, which was carried out in an outpatient psychiatry clinic. The study reported that women in polygamous settings were less educated than those in monogamous settings and among men, those in polygamous setting had lower educational attainment [9]. In this study, respondents in both marriage settings were predominantly Muslims $(100 \%)$ and this is probably due to the fact that the study area (Sokoto state) is located in North West geo-political zone, a zone which is predominantly inhabited by Muslims [16].

The overall prevalence of depression (in both monogamous and polygamous groups) in this study was $52.1 \%$. This prevalence is quite high, however, it is lower than the prevalence rate of $59.6 \%$ reported by Afolabi et. al in Osogbo southwest Nigeria [17]. The prevalence of depression in this study is nevertheless, high when compared to the prevalence of depression observed in a study on depressive disorders among pregnant women in Sagamu, south-west Nigeria [18], which reported prevalence of $29.1 \%$. The lower prevalence observed in the study in Sagamu could be attributed to the fact that the study was hospital based as against our study, which was community based, thus more likely to detect hidden cases of depression within the community. Our findings may follow the "Iceberg Phenomenon" of disease, in which only the symptomatic cases are seen in the hospital, the vast majority (mainly asymptomatic cases) remain undetected within the community [19].

Out of the $52.1 \%$ of the respondents that had depression, $28 \%$ were those married in monogamous setting, which is slightly higher than the prevalence rate among those married in polygamous setting $(24.1 \%)$. This finding is contrary to the findings of a study conducted in Turkey which reported a lower prevalence of depression among women in monogamous setting (18\%), however, in the same study in Turkey, the prevalence rate among those married in polygamous setting was $40 \%$, which is much higher than the $24.1 \%$ observed in this study [6]. Another study conducted in north-central Nigeria also observed similar pattern where prevalence of depression was higher among women married in polygamous setting than in monogamous setting [20]. The lower prevalence observed in our study among those in polygamous marriages may not be unrelated to the fact that polygamous marriages are highest in the northwest region of Nigeria [21] (47.5\%), thus making the women in this part of the country probably better adapted to marriages in polygamous settings than women in Turkey; moreover, polygamous marriage is illegal in Turkey [22], therefore, women who finds themselves in such relationship may likely be more prone to depression.

On the pattern of depression observed in this study, overall prevalence of mild depression in both groups was $25.2 \%$, however, among those in monogamous setting, the proportion of those with mild depression was $43.1 \%$ whereas among those in polygamous setting, the proportion with mild depression was $54.3 \%$. The overall prevalence of mild depression $(25.2 \%)$ observed in this study is low when compared to the $42.8 \%$ reported by Afolabi et al in a study on the pattern of depression among patients in a Nigerian family practice clinic [17]. A much higher prevalence of mild depression was reported in a study on prevalence of depression in a Primary Health Care setting in North Central Nigeria which was as high as $84.9 \%$ [20]. Reasons for the disparity could be explained by the fact that different denominators were used between this study and the other two 
studies; in our study, the denominator constituted all the respondents $(\mathrm{N}=378)$ whereas the other studies looked at the prevalence of mild depression among those with depression.

Overall prevalence of moderate depression was $14.8 \%$ out of which the proportion among those in monogamous setting was $34.9 \%$ and $21.3 \%$ among those in polygamous setting. This finding (14.8\% prevalence of moderate depression) is similar to the findings of Sanni et al [20] who observed a prevalence of $14.2 \%$ in Illorin, Nigeria, however, a slightly higher prevalence of moderate depression (16\%) was reported by Afolabi et al in their study from Osogbo, southwest Nigeria [17]. In a study conducted in the United States of Americaby Dolittle and Farrel [23], a much higher prevalence of moderate depression $(22 \%)$ was observed; the differences between the observed prevalence in this study and the values reported in other studies both within and outside Nigeria may reflect a variation in local factors influencing depression in the various communities, as has also been suggested by Judd et al [24]. The overall prevalence of severe depression was $3.6 \%$, however, the proportion among women in monogamous setting was $4.6 \%$ while among those married in polygamous setting, the proportion with severe depression was $9.6 \%$. This finding is higher than the prevalence of severe depression $(0.94 \%)$ observed in a psychiatric clinic in North Central Nigeria [20].

Among women in polygamous settings, there was a statistically significant association between dispute with co-wife and depression. This finding is consistent with that of Al-krenawi which was carried out among women from monogamous and polygamous marriages in an outpatient psychiatric clinic in Ar-Raqqah, north-central Syria, which reported a statistically significant association between dispute among co-wives and depression [9]. Among the reasons given by women married in polygamous settings for their sadness were poor welfare from the husband, uneven distribution of resources among co-wives by husband, jealousy, marital insecurity and dispute between co-wives. These findings are in keeping with the findings of Al-Krenawi, which attributed depression in polygamous setting to jealousy, uneven distribution of resources and competition among co-wives [9]. Even though this study did not look at pattern of depression among co-wives, especially between $1^{\text {st }}$ wife and other co-wives, studies conducted in Turkey and Egypt found that senior wives in polygamous families experienced a major psychological crisis, which manifests itself in somatic complaints as well as in psychological symptoms such as anxiety, depression and irritability following their husbands' second marriage [6, $10]$.

On bivariate analysis, factors associated with depression were marital status, being sad for a long time and having family history of depression. On multivariate regression analysis however, significant predictors of depression were marital status and being sad for a long time. In this study, respondents who were married were found to be about four times less likely to develop depression in both groups. In the study from the north-central part of Nigeria it was reported that being married or the type of marriage did not confer protection against depression. It is believed that marriage in itself does not does not confer any protect against depression but rather it is the quality of emotional and social support that married partners derive from each other that confers protection [20]. Although family history of depression was found to be associated with depression among women in monogamous setting, it was not found to be a significant predictor of depression. In a related study conducted in Slovenia, Zalar et al opined that "no evidence of a link between the presence of putative high risk alleles and the likelihood of either having depression or having a family history of depressive disorder" [25]. In a systematic review on prevalence and correlates of depression among Australian women by Rich et al [26], family history of mental illness was found to be a significant correlate of depression. These conflicting findings regarding possible link of depression and family history probably suggests that social rather than biological factors have more influence on depression. Other studies on possible link of some genetic factors and development of major depressive disorders also found conflicting results $[27,28]$.

\section{Limitations of the Research}

A major limitation of this study was the paucity of researches pertaining the prevalence and pattern of depression in relation to family setting (monogamous and polygamous), thus most of the findings of this study were compared with studies on the prevalence and pattern of depression among the general population. Also, this study did not look at pattern of depression among co-wives in polygamous families, thus there is need for further research in this area, especially in north-west Nigeria, where the prevalence of polygamous marriage is very high.

\section{Conclusion and Recommendation}

The overall prevalence of depression was high in this study and the prevalence was slightly higher in monogamous group than polygamous marriages; most respondents in both groups had mild depression. Being sad for most of the days was among the major symptoms experienced by respondents and part of the reasons given by respondents for their sadness included poor welfare from the husband, uneven distribution of resources among co-wives by husband, jealousy, marital insecurity and dispute between co-wives. Significant predictors of depression include marital status and being sad for long time. Given the high prevalence of depression in this study, there is need for government and other partners to strengthen mental health services at community levels to enable early detection and management of depression among couples. There is also need for further research to look at pattern of depression among co-wives in polygamous family settings, especially in north-west Nigeria, where the prevalence of polygamous marriage is very high. 


\section{Contribution of Authors}

Adamu $\mathrm{H}$ and Oche MO conceived the initial idea for the manuscript. Abel RH, Garba KA and Zubairu BB took part in the design of the questionnaire, the data collection, analysis and wrote the first draft of the manuscript under the supervision and guidance of Adamu $\mathrm{H}$ and Oche MO. All the authors contributed to the revision of the manuscript and approved the final manuscript.

\section{Conflict of Interest}

The authors declare that they have no competing interests.

\section{References}

[1] Jack-Ide I. A Retrospective study of the prevalence of depression among women attending a mental health clinic in the Niger Delta Region of Nigeria. Annals of Behavioral Science 2016; 2 (1): 12.

[2] American Psychiatric Association. Depression. Available at: https://www.psychiatry.org.

[3] World Health Organization. Depression: Facts and figures. 2018 (cited 25 Sept 2019) Available at: https://www.who.int/newsroom/fact/detail/depression.

[4] Shittu RO, Odeigah LO, Issa BA, Olanrewaju GT. Association between depression and social demographic factors in a Nigerian family practice setting. Open Journal of Depression 2014; 3 (1): 18-23.

[5] Bautista CF, Velázquez JV, Icaza MEMM, López MM, Gutiérrez López Mde L, et al. Socio-demographic and personal factors related to depressive symptomatology in the mexican population aged 12 to 65. Rev Bras Psiquiatr 2012; 34 (4): 395-404.

[6] Ozkan M, Altindag A, Oto R, Sentunali E. Mental health aspects of Turkish women from polygamous versus monogamous families. International Journal of Social Psychiatry 2006; 52 (3): 214-20.

[7] Rondet C, Cornet P, Kaoutar B, Lebas J, Chauvin P. Depression prevalence and primary care among vulnerable patients at a free outpatient clinic in Paris, France, in 2010: results of a cross-sectional survey. BMC Fam Pract 2013; 14 (1): $151-159$.

[8] Al Krenawi A, Graham J. A comparison of Family Functioning, Life and Marital Satisfaction and Mental health of Women in Polygamous and Monogamous Marriage. International Journal on Social Psychiatry 2006; 52 (1): 5-17. Available from: (https://www.ncbi.nlm.nih.gov).

[9] Al-Krenawi A. Women from polygamous and monogamous marriages in an outpatient clinic in Negev. Transcultural Psychiatry 2001; 38 (2): 187-199 (cited Sept 2019) Available at: (https://www.tps.sage.pvb.com).

[10] Al-Sherbiny LAM. The case of first wife in polygamy: Description of an Arab culture-specific tradition. Arabpsynet 2005; 8: 9-26.

[11] Kam K. Depression and Risky behavior. Web MD June 2011 (cited 25 Sept 2019) Available at: (https://www.webmd. com).

[12] Bodinga Local Government Area Nigeria. Available at: https://citypopulation.de/php/nigeria-admin.php?adm2id=NG A034002.

[13] Ibrahim T. Research methodology and Dissertation writing; Abuja, Crest Global Links LTD 2009: 118.

[14] Agarwal P, Halle Z, Sambarmoorthi U. Prevalence of Depression in a West African Country: Evidence from World Health Survey. 2013. Elsevier (cited 25 Sept 2019). Available at: (https://www.valueinhealthjournal.com).

[15] Halverson JL, Bienenfeld D. What are the DSMS criteria for diagnosis of major depressive disorder? 2019 Available at: https://www.medscape.com/answers/286759-14692/what-are-t he-dsm-5-criteria-for-diagnosis-of-major-depressive-disorderclinical-depression.

[16] National Population Commission (NPC) [Nigeria] and ICF. 2019. Nigeria Demographic and Health Survey 2018 Key Indicators Report. Abuja, Nigeria, and Rockville, Maryland, USA: NPC and ICF.

[17] Afolabi M, Abioye-Kuteyi E, Faloye F, Bello I, Adewuye A. Pattern of depression among patients in a Nigerian family practice population. SA Fam Pract 2008; 50 (2); 63.

[18] Ogunsemi OO, Oluwole FA, Abasiubong F, Erinfolami AR, Amoran OE, Ariba AJ et al. Detection of mental disorders with the patient health questionnaire in primary care settings in Nigeria. Ment Illn 2010; 2: 46-50.

[19] Park K. Park's textbook of preventive and social medicine, 21st Edition: Banarsidas Bhanot, India 2011.

[20] Sanni O, Onuoha F, Alabi A, Ayinmode A, Buhari O. Epidemiology of Depression in a Primary Care Setting in North Central Nigeria. Global Journal of Endocrinological $\begin{array}{llll}\text { Metabolism } & 2018 ; & 2 & \text { (4). }\end{array}$ 10.31031/GJEM.2018.02.000542. Available at https://crimsonpublishers.com/gjem/fulltext/GJEM.000542.ph p.

[21] National Bureau of Statistics (NBS) and United Nations Children's Fund (UNICEF). 2018 Multiple Indicator Cluster Survey 2016-17, Final Report. Abuja, Nigeria: National Bureau of Statistics and United Nations Children's Fund.

[22] Ekerbiçer HC, Resim S, Efe E, Bahar MR, Altunören Ozlem, Küçükdurmaz $\mathrm{F}$ et al. A Comparison of Sexual Function, Psychological Status, and Sociodemographic Characteristics of Turkish Men within Polygamous and Monogamous Marriages. Balkan Med J. 2016 Jul; 33 (4): 383-389.

[23] Dolittle R, Farrell M. The association between spirituality and depression in an urban clinic. J Clin Psych 2004; 6: 114-8.

[24] Judd FK, Jackson HJ, Komiti A, Murray G, Hodgins G, Fraser C. High prevalence disorders in urban and rural communities. Aust N Z J Psychiatry 2002; 36 (1): 104-7.

[25] Zalar B, Blatnik A, Maver A, Klemenc-Ketiš Z, and Peterlin B. Family History As an Important Factor for Stratifying Participants in Genetic Studies of Major Depression. Balkan J Med Genet. 2018; 21 (1): 5-12. 
[26] Rich JL, Byre JM, Curryer C, Byles JE and Loxton D. Prevalence and correlates of depression among Australian women: a systematic literature review from Jan 1999-Jan 2010. BMC Research Notes. 2013; 6: 424. Available at http://www.biomedcentral.com/1756-0500/6/424.

[27] Cohen-Woods S, Craig IW, McGuffin P. The current state of play on the molecular genetics of depression. Psychol Med. 2013; 43 (4): 673 [PubMed] [Google Scholar].

[28] Bosker FJ, Hartman CA, Nolte IM, Prins BP, Terpstra P, Posthuma D. et al. Poor replication of candidate genes for major depressive disorder using genome-wide association data. Mol Psychiatry. 2011; 16 (5): 516. 